\title{
Experimental verification on applying indirect inverse substructuring analysis to identify coupling dynamic stiffness of mechanical assembly via planar surface
}

\author{
Guang-qing Lu ${ }^{1}$, Kun-peng $\mathrm{Xu}^{2}$, Wen-bin Peng ${ }^{3}$, Minvydas Ragulskis ${ }^{4}$, Bo Wang ${ }^{5}$ \\ 1, 2,3,5School of Electrical and Information Engineering, Jinan University, Zhuhai 519070, China \\ ${ }^{2}$ School of Mechanics and Construction Engineering, Jinan University, Guangzhou 510632, China \\ ${ }^{4}$ Department of Mathematical Modelling, Kaunas University of Technology, Kaunas 50282, Lithuania \\ ${ }^{1}$ Corresponding author \\ E-mail: ${ }^{1}$ tgqluyp@jnu.edu.cn, ${ }^{2} x u k p @ s t u 2017 . j n u . e d u . c n,{ }^{3} 010943243 @ q q . c o m$, \\ ${ }^{4}$ minvydas.ragulskis@ktu.lt, ${ }^{5}$ twb@jnu.edu.cn
}

Received 1 October 2018; accepted 8 October 2018

DOI https://doi.org/10.21595/vp.2018.20299

Check for updates

Copyright $(C 2018$ Guang-qing Lu, et al. This is an open access article distributed under the Creative Commons Attribution License, which permits unrestricted use, distribution, and reproduction in any medium, provided the original work is properly cited.

\begin{abstract}
To broaden the engineering application of inverse substructuring analysis, the mechanical assembly via planar surface is experimentally studied. Specifically, the first and the second schemes of indirect inverse substructuring analysis are applied to identify the coupling dynamic stiffness of the assembly. The experimental model of the assembly is designed, and the surface is then discretized equivalently into point-to-point connections for testing the frequency response functions (FRFs) involved in the schemes. Experimental results show that, applying both of the schemes are feasible for the identification, and the identified stiffnesses approach to be stable as the number of discretized points increases.
\end{abstract}

Keywords: indirect inverse substructuring analysis, coupling dynamic stiffness, mechanical, assembly, planar surface.

\section{Introduction}

In general, an electro-mechanical product consists of parts or components via mechanical assemblies. Quality inspection of an assembly considers tolerance and or size-coordination conventionally [1]. However, the dynamic quality of the involved assemblies should be also inspected in consideration of dynamic quality of a whole product [2]. Dynamic quality of a product is affected seriously by the dynamic quality of its mechanical assemblies. The dynamic quality of an assembly can be estimated via the dynamic characteristics of the coupling interface or joint connection(s) involved the assembly [3]. Identifying the joint-properties is complicated via conventional 'positive' methods $[4,5]$, which belong to dynamic substructuring analysis and result in low accuracy in engineering application.

Applying FRF-based inverse substructuring dynamic analysis is considered as the effective method determine the dynamic characteristics of joint or connection(s) of a mechanical assembly [6]. It has been applied to fix the eigenvalue of an assembly matrix [7]. In addition, due to the operational difficulty in testing the FRFs involved in the direct scheme of inverse substructuring analysis provided in literature [6], Lu Guangqing developed five indirect schemes of inverse analysis for identifying the coupling dynamic stiffness of a mechanical assembly [8] and vibrational structures [9]. The stiffness and eigenvalues can be used as two quantitative criterions of dynamic quality of a mechanical assembly. The theoretical completeness of the indirect schemes was validated analytically by simulations, and their feasibility and effectiveness in application in case of ideal coupling interface with point-to-point connection of mechanical assembly were also verified by experiments in literatures $[8,9]$.

This study applies the first and the second indirect schemes of inverse substructuring analysis of literature [8] to identify the stiffness of mechanical assembly with non-ideal coupling interface. An experimental model of the assembly via planar surface is designed, and the surface is 
equivalently discretized into a number of 'point-to-point connections' in order to meet the essential needs of the indirect schemes. Then, the FRFs involved are tested on the model, and the stiffnesses are computed by the schemes. The feasibility of applying the schemes is verified experimentally in case of the assembly via planar surface.

\section{The indirect schemes to identify the coupling dynamic stiffness}

In general, a mechanical assembly system with discrete couplings can be equivalently described by the model of two-level substructures with 'substructure/component A-coupling connector-substructure/component B', as shown in Fig. 1. The discrete couplings are denoted by $c a$ and $c b$, and the external input of exciting dynamic force on component $\mathrm{B}$ and the output response of dynamic displacement on component $\mathrm{A}$ are denoted by $i b$ and $o a$. The 'FRF at system level' of the assembly system is denoted by $H_{S}$, and the 'FRF at component level' of A and B are denoted by $H_{A}$ and $H_{B}$, respectively. The coupling dynamic stiffness of the connector is represented as $K_{s}$.

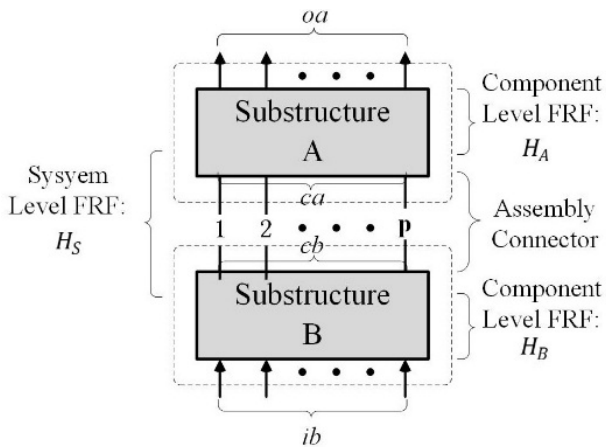

Fig. 1. Model of two-level substructures with discrete couplings

If choosing the same numbers of $(i a, i b),(o a, o b)$ and $(c a, c b)$, then five FRF-based indirect schemes of inverse substructuring analysis for determining coupling dynamic stiffness matrix, $\boldsymbol{K}_{s}$, of connector can be derived [8]. Where, the first and second schemes are expressed as:

$\boldsymbol{K}_{s}=\left[H_{\text {cbibHS,oaib }}^{-1} \boldsymbol{H}_{\text {oaca }}-\boldsymbol{D}\right]^{-1}$ (first indirect scheme),

$\boldsymbol{K} s=\left[H_{\text {caia }}\left(\boldsymbol{H}_{\text {oaia }}-H_{s, \text { oaia }}\right)-1 \boldsymbol{H}_{\text {oaca }}-\boldsymbol{D}\right]-1$ (second indirect scheme),

where, $\boldsymbol{D}=\boldsymbol{H}_{c a c a}+\boldsymbol{H}_{c b c b}$. Eqs. (1) and (2) show that testing the FRFs at system level on the coupling interfaces in assembling state is unnecessary. And using Eq. (1) and Eq. (2) to identify the stiffness need 5 and 6 FRF-matrices, respectively. For the first scheme, four FRFs at component level before assembly, $\boldsymbol{H}_{\text {oaca }}, \boldsymbol{H}_{c b i b}, \boldsymbol{H}_{c a c a}$ and $\boldsymbol{H}_{c b c b}$, and one FRF at system level after assembly, $\boldsymbol{H}_{s, \text { oaib }}$, are to be tested. For the second scheme, five FRFs at component level, $\boldsymbol{H}_{\text {caia }}, \boldsymbol{H}_{\text {oaia }}, \boldsymbol{H}_{\text {oaca }}, \boldsymbol{H}_{\text {caca }}$ and $\boldsymbol{H}_{c b c b}$, and one FRF at system level, $\boldsymbol{H}_{s, c a i a}$, are to be tested. All the FRFs are shown in Fig. 2.

\section{Experimental model of mechanical assembly via planar surface}

To verify the feasibility of applying both the first and the second indirect schemes to identify the coupling dynamic stiffness matrix, $\boldsymbol{K}_{s}$, of mechanical assembly via planar surface, here designs an experimental model of the assembly with the connection of planar interface, as shown in Fig. 3. Components A and B consist of a steel plate $\left(300 \times 260 \times 10 \mathrm{~mm}^{3}\right)$ attached with four different mass of steel blocks by damping rubber cylinders ( $\Phi 35 \mathrm{~mm})$, as shown in Fig. 3(a)-(c). They are assembled together via an ordinary hard rubber layer (3mm thick) as the planar 
connector, see Fig. 3(d). The discretized coupling points (No.1-8) and the positions of the blocks (No.1-4) are shown in Fig. 3(e).

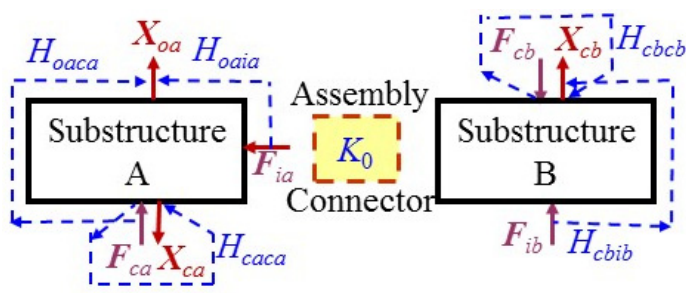

a) FRF at component level for the indirect schemes
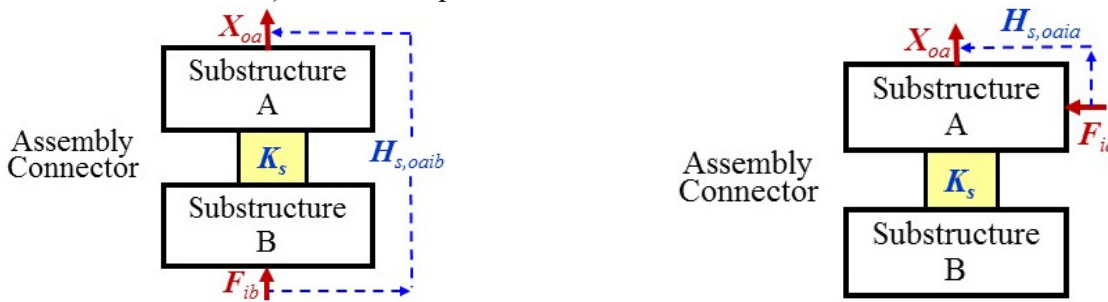

b) FRF at system level for the indirect schemes c) FRF at system level for the second indirect schemes

Fig. 2. The tested FRFs for the first and the second indirect schemes

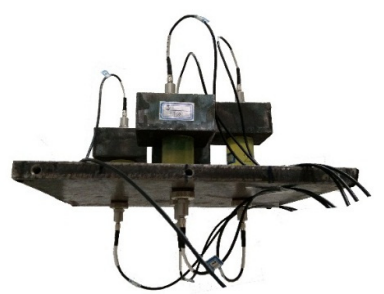

a) Component $\mathrm{A}$

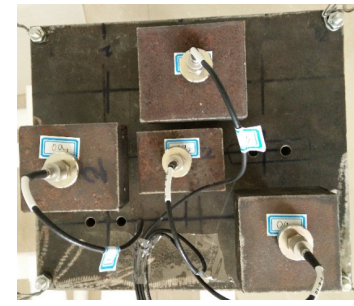

b) Vertical view

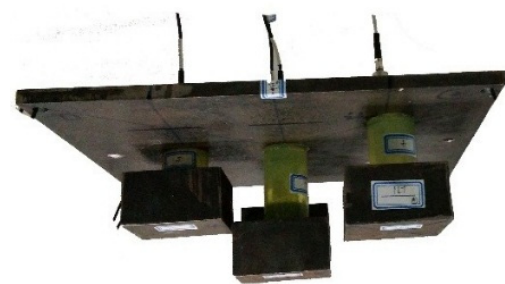

c) Component B

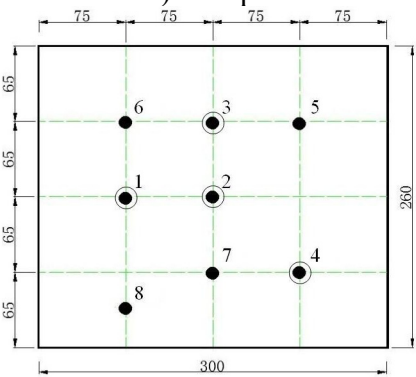

e) Discrete points

d) Assembling system

Fig. 3. Experimental model of mechanical assembly with linear connection

Because the FRFs involved in the indirect schemes of Eqs. (1) and (2) are required to be tested by "point-viro-excitation to point-displacement-response" on either the components A and B or their connection of planar surface, the surface should be discretized into a number of 'point-to-point connections', theoretically, there are infinite number of the discrete points. In order to check if it is valid to use limited discrete number of points instead of the infinite number of points equivalently, the planar surface is divided uniformly in grid, as shown in Fig. 3(e). Correspondingly, there are bi-, tri-, quad-, pent-, hexa-, hepta- and octa-coupled 'point-to-point connections' on the surface. By vibro-excitation tests, all the FRF-matrices of Eqs. (1) and (2) are acquired respectively in these seven cases of experimental models with discrete "point-to-point connections" under free state. The testing frequency is set to be $0-50 \mathrm{~Hz}$ in this study. 


\section{Experimental results}

All the entries of the five and six square FRF-matrices involved in the first and second indirect schemes in the seven cases of "point-to-point connections" are tested firstly on the experimental model of mechanical assembly via planar surface, as shown in Fig. 3, the coupling dynamic stiffness matrix $\boldsymbol{K}_{s}$ in each case is computed by Eq.(1) and Eq.(2) for the first and second scheme, respectively. The mean module spectra, $\left|K_{s}(f)\right|$, of diagonal entries of the matrix, $K_{\text {sii }}$ ( $i=1,2, \ldots, p$, and $p=2-8$ is the number of discrete points), in the six cases are plotted in Fig. 4 . Fig. 5 shows their amplified plots in range of $20-30 \mathrm{~Hz}$.

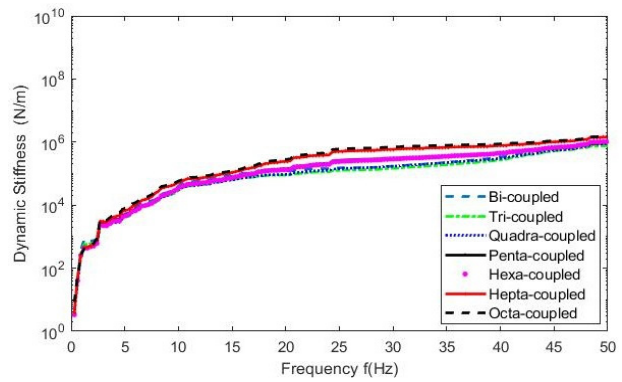

a) First indirect scheme

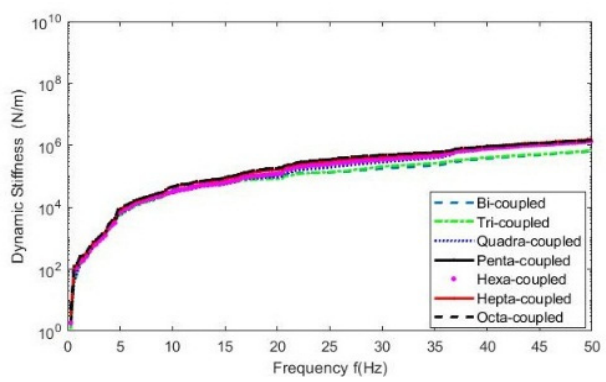

b) Second indirect scheme

Fig. 4. Mean $\left|K_{s}(f)\right|$ in testing frequency range in six cases of discrete points

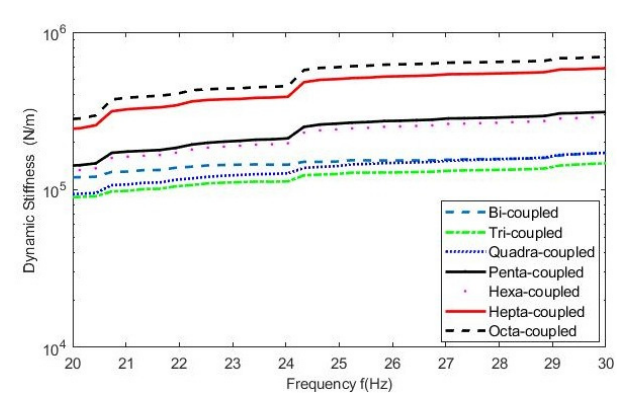

a) First indirect scheme

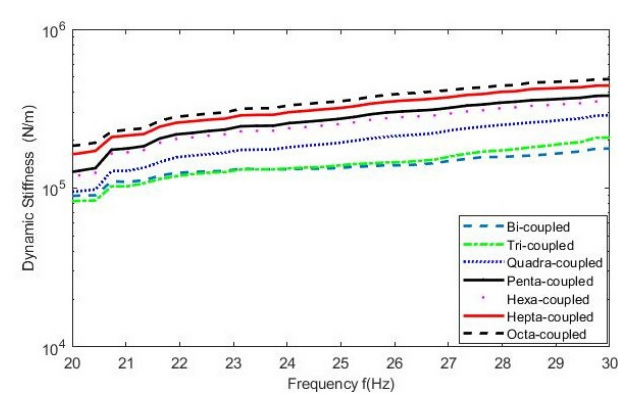

b) Second indirect scheme

Fig. 5. Amplified mean $\left|K_{S}(f)\right|$ in $20-30 \mathrm{~Hz}$ in the seven cases of discrete points

To show the differences of the identified stiffnesses, here takes the mean $\left|K_{s}(f)\right|$ in case of octa-coupled connection as a reference, denoted by $\left|K_{m}(f)\right|$. The relative errors, $E_{m i}$, $(i=1,2, \ldots, 6)$ of the first six cases in full testing frequency range $(0-50 \mathrm{~Hz})$ are computed by:

$E_{m i}=\frac{\sum_{n=1}^{N}|| K_{s i}\left(f_{n}\right)|-| K_{m}\left(f_{n}\right)||}{\sum_{n=1}^{N}\left|K_{m}\left(f_{n}\right)\right|} \times 100 \%$

where $N=400$ is the number of discrete frequencies. The computed $E_{m i}$ are listed in Table 1 .

Table 1. Relative errors $E_{m}(\%)$ of mean $\left|K_{s}(f)\right|$ in six cases to $\left|K_{m}(f)\right|$ in case of octa-coupled connection

\begin{tabular}{|c|c|c|c|c|c|c|}
\hline \multirow{2}{*}{ Indirect scheme } & \multicolumn{6}{|c|}{ Case of point-to-point connections } \\
\cline { 2 - 7 } & Bi-coupled & Tri-coupled & Quad-coupled & Pent-coupled & Hexa-coupled & Hepta-coupled \\
\hline First & 11.0 & 10.7 & 8.6 & 6.1 & 4.6 & 3.4 \\
\hline Second & 8.5 & 6.7 & 5.5 & 6.0 & 4.4 & 1.4 \\
\hline
\end{tabular}

It can be seen from Figs. 3, 4 and Table 1 that:

(1) The identified stiffnesses in the seven cases of discrete point numbers are on the same order of magnitude, which is around $10^{4}-10^{6} \mathrm{~N} / \mathrm{m}$ and on practical order of hard rubber stiffness, as 
shown in Fig. 3. This verifies that discretizing the coupling interface of planar surface into "point-to-point connections" equivalently and applying the indirect scheme to identify the stiffness of mechanical assembly is feasible.

(2) Fig. 4 shows that the identified stiffness draws close to be stable with increase of the number of discretized points.

(3) Table 1 shows that, for the experimental model in this study, discretizing its planar surface into equivalent "point-to-point connections" results in closer mean values of identified stiffness, subsequently, smaller relative errors to the maximum number of discrete points (in case of octa-coupled connection). This is also demonstrated in Fig. 5. Implicitly, there probably exists an maximum number of discrete points for a specific mechanical assembly that can be approximately considered as the optimum number for discretizing the planar surface of assembly, instead of infinite number of discrete points.

(4) The second indirect scheme shows better performance than the first indirect scheme on discretizing the planar surface of a mechanical assembly, due to closer results of identified stiffness with increase of discrete points.

\section{Conclusions}

This study applies both the first and the second indirect schemes of inverse substructuring analysis to identify the coupling dynamic stiffness of mechanical assembly via planar surface, an experimental model is designed, and the surface is equivalently discretized into "point-to-point connections" in seven cases at first. Then, the stiffness is computed by the schemes using the FRF-matrices tested by vibro-excitation. As the results of the experimental study, the schemes are firstly verified to be feasible in engineering application to the assembly via planar surface as a connector. With increase of the number of discretized points, the identified stiffness approaches to a stable value. It seems that the second indirect scheme has better performance on discretizing the surface. This means implicitly that there probably exist definite discrete points of the surface for specific assembly to apply equivalently the schemes, instead of infinite number of discrete points.

\section{Acknowledgements}

The authors acknowledge gratefully National Natural Science Foundation of China for supporting the Project (Grant No. 51475211), and Guangdong Provincial Department of Science and Technology for supporting the Provincial-ministerial University-industry Cooperation Project (Grant No. 2013B090600142).

\section{References}

[1] Wang Bo, Tang Xiaoqing Decision-making of quality control for mechanical assembly activities. China Mechanical Engineering, Vol. 21, Issues 2-2010, 164, p. 168-174, (in Chinese).

[2] Lu Guangqing, Yi Chuijie, Fang Ke Analysis and inverse substructuring computation on dynamic quality of mechanical assembly. Chinese Journal of Mechanical Engineering, Vol. 29, Issue 3, 2016, p. 539-548.

[3] Lu Guangqing, Fang Ke, Pang Dongmei Substructuring analysis and quantitative computation on dynamic quality of mechanical assembly. Mechanical Science and Technology for Aerospace Engineering, Vol. 35, Issue 6, 2016, p. 840-845, (in Chinese).

[4] Damjan C., Miha B. Identification of the dynamic properties of joints using frequency response functions. Journal of Sound and Vibration, Vol. 317, Issues 1-2, 2008, p. 158-174.

[5] Majid M., Graham E., Park S. S. FRF based joint dynamics modeling and identification. Mechanical Systems and Signal Processing, Vol. 39, Issues 1-2, 2013, p. 265-279.

[6] Zhen J. T., Lim T. C., Lu G. Q. Determination of system vibratory response characteristics applying a spectral-based sub-structuring approach, Part I: Analytical formulation. International Journal of Vehicle Noise and Vibration, Vol. 1, Issues 1-2, 2004, p. 1-30. 
EXPERIMENTAL VERIFICATION ON APPLYING INDIRECT INVERSE SUBSTRUCTURING ANALYSIS TO IDENTIFY COUPLING DYNAMIC STIFFNESS OF MECHANICAL ASSEMBLY VIA PLANAR SURFACE. GUANG-QING LU, KuN-PENG XU, WEN-BIN PENG, MinVYDAs RAGULSKIS, Bo WANG

[7] Guang-qing Lu, Ke Fang, Dong-mei Pang Eigenvalue analysis and estimation on dynamic quality of mechanical assembly. Journal of Vibroengineering, Vol. 17, Issue 8, 2015, p. 4390-4403.

[8] Lu Guangqing, Yi Chuijie, Fang Ke Inverse substructuring methods for identifying coupling dynamic stiffness of mechanical assembly. Journal of Mechanical Engineering, Vol. 52, Issue 9, 2016, p. 86-95, (in Chinese).

[9] Lu Guangqing, Wang Minqin, Wang Bo, et al. Indirect inverse substructuring identification method for coupling dynamic stiffness of vibrational structures. Chinese Journal of Acoustics, Vol. 37, Issue 2, 2018, p. $241-256$ 